\title{
Episiotomy and operative vaginal delivery- Do we need more evidence?
}

\author{
Abdul Sultan ${ }^{1}$ and Jan Willem de Leeuw ${ }^{2}$ \\ ${ }^{1}$ Croydon University Hospital \\ ${ }^{2}$ Ikazia Hospital
}

April 7, 2021

\section{Episiotomy and operative vaginal delivery- Do we need more evidence?}

\author{
A.H. Sultan
}

- Urogynaecology and Pelvic Floor Reconstruction Unit, Croydon University Hospital, London Road, Croydon CR7 7YE

- Honorary Reader, St George's University of London

Email:asultan29@gmail.com

Tel: 00447961386840

(ORCID 0000-0001-8979-2304)

J.W. de Leeuw, Department of Obstetrics and Gynaecology, Ikazia Ziekenhuis, Rotterdam, the Netherlands

(ORCID 0000-0001-5028-8055)

\section{DISCLOSURE of INTEREST}

Abdul Sultan is the co-director of the Croydon Perineal and Anal Sphincter Trauma courses (www.perineum.net)

Operative vaginal delivery (OVD) is recognised as a major risk factor in the occurrence of obstetric anal sphincter injuries (OASIs), particularly during first vaginal deliveries. Randomised controlled trials (RCTs) have shown the merits of adopting a policy of restrictive mediolateral episiotomy during normal vaginal delivery, although no RCT to date has included measurements of the angle or size of the episiotomy. The benefits of episiotomy performed during OVD demonstrated in large observational studies are overwhelming (Sultan et al. Eur J Obstet Gynecol Reprod Biol. 2019;240:192-196) .

Ankarcrona et al have added another study to this collection and have confirmed the results of most such publications. In their study, based on 11 years of data from the Swedish Medical Birth Register, they have emulated a RCT using propensity scores. Ultimately, both methods used showed an almost identical risk reducing effect as the commonly used logistic regression analysis. demonstrating a significant reduction in OASIs during vacuum extraction associated with the use of mediolateral or lateral episiotomies. The Number Needed to Treat to prevent one OASI was 27 , which is known to be fourfold lower in forceps delivery.

Is the episiotomy a treatment for a certain condition or disease? In reality, episiotomy is an intervention to reduce the risk for an unwanted side effect of birth. Consequently, the impact is one of risk modification as opposed to treatment. Similar to the study by Ankarcrona et al risk factors are commonly established with the use of observational studies (RCOG Greentop guideline No 29, 2015). In the last decade, several 
large observational studies Involving more than 2 million women showed a significantly lower rate of OASI in nulliparous women undergoing OVD with an episiotomy.

Given the availability of such studies, based on registered databases, showing significantly lower OASI rates, is there still a need for further evidence? Ankarcrona et al acknowledge Lund et al who have shown in their systematic review that there is an association between the risk reduction for OASI with episiotomy rates; the greatest reduction was shown in studies with episiotomy rates over $70 \%$.

Obstetricians opposing the use of routine episiotomy during OVD highlight the lack of a definitive RCT. RCT's are commonly used to address the treatment effect of an intervention on a particular condition with a well described outcome. However, RCT's of episiotomy during OVD have proven to be very difficult and usually compare no more than the liberal versus the restricted use of episiotomy. As Ankarcrona et al mention, there is only one pilot RCT of IVD and episiotomy indicating that 1600 OVD will need to be included for a definitive study. However, we believe that the design of such a study should be two separate arms for forceps and vacuum delivery as the inherent risks with/without an episiotomy is different. Such a study with vacuum extraction is currently underway in Sweden.

The challenge now is to identify prior to labour which women are at high risk of sustaining OASIS using prediction models based on the pre-existing large national databases. 